\title{
Info-Chunk Objects as New Behavior Representation for System-based Model of Product
}

\author{
Yatish Bathla
}

Doctoral School of Applied Informatics and Applied Mathematics, Óbuda University, Bécsi út 96/b, H-1034 Budapest, Hungary

yatish.bathla@phd.uni-obuda.hu

Abstract: Requirement Functional Logical Physical (RFLP) structure has emerged as one of the prominent approaches for modeling the multidisciplinary products. Information Content (IC) provides effective interaction between the human and multidisciplinary product model. Though it controls the RFLP level by the Multilevel Abstraction based SelfAdaptive Definition (MAAD) structure, it needs to be further enhanced in terms of HumanComputer Interaction (HCI), multidisciplinary product behavior representation and structured processing of interrelated engineering objects to obtain coordinated decisions. Therefore, this paper introduces the Object-Oriented Principle $(O O P)$ concepts in the IC for behaviors representation of the multidisciplinary product where Info-Chunk is considered as an object. Here, Behavior Info-Chunk (BiC) and Context Info-Chunk (CxiC) objects are proposed in the MAAD structure to model the behavior of the multidisciplinary product. Further, the concepts of Info-Chunk objects are extended to Intelligent Property (IP) that uses Initiative Behavior Context and Action (IBCA) structure to handle the RFLP structure. Based on the communication between the MAAD and RFLP structure, an API (Application Programming Interface) called "InfoChunkLib" is proposed. It can generate the graphs to represent the behaviors of a multidisciplinary product model. The API is handled by the information content to represent the behavior information and store the results in a database.

Keywords: Behaviors representation; Multidisciplinary product modeling; Info-Chunk based Information Content; RFLP structure; MAAD structure; IBCA structure

\section{Introduction}

Modeling of multidisciplinary products requires coordination of a significant amount of model information. The integrated definition is raised to the conceptual level of product design, which requires high-level abstraction. A four-leveled structure of the product model using Requirement Functional Logical Physical 
(RFLP) structure [2] was introduced in the virtual environment. It is applied from system engineering and offers handling product and its model as a system. It accommodates product behavior definitions on its $\mathrm{F}$ and $\mathrm{L}$ levels. Product assembly is done in the specification tree (red square) of RFLP structure as shown in Fig. 1. Due to complex Human-Computer Interaction (HCI), Information Content (IC) was used to record and apply the content of information that is represented in the product model space [19]. In this content, an intent is defined by the human to control the definition of engineering objects [22]. IC [1] controls the RFLP level by the Multilevel Abstraction based Self-Adaptive Definition (MAAD) structure [2]. However, IC needs to be enhanced in terms of the practical feasibility of HCI, behavior representation and structured processing of interrelated engineering objects to obtain the coordinated decisions. To solve above-mentioned issues, this research work proposes the Info-Chunk objects and InfoChunkLib API (Application Programming Interface) is proposed in the IC.

Info-Chunk objects are based on the Object-Oriented Principle (OOP) concepts that are used in software programming. Previous research work deployed OOP concepts in the RFLP structure in the form of the Modelica language [6]. It is used for logical and physical modeling of a multidisciplinary product. Here, models and their components are defined by the object diagram. This research work uses OOP concepts in the Functional layer and Logical layer of the RFLP structure for behaviors representation of the multidisciplinary product modeling. Here, InfoChunk entity is converted into the object first. Then, Behavior Info-Chunk (BiC) object and Context Info-Chunk (CxiC) object are introduced in the MAAD structure and the IBCA structure to store the behaviors of the multidisciplinary product. The proposed Info-Chunk objects are used to establish a link with the Layer Info-Chunk (LiC) objects of RFLP structure. InfoChunkLib API is coded based on the communication between the MAAD structure and RFLP structure. The Java language is used as a JavaFX application. It represents the behaviors of the components in the multidisciplinary product model. The generated output is shown using the graph between the components of engineering disciplines. IC imports the InfoChunkLib API and coded as a Web application. The multidisciplinary product model can be more efficiently handled through the IC instead of the Specification Tree. This paper begins with the conversion of InfoChunk entity into the object. Further, behavior Info-Chunk (BiC) objects and context Info-Chunk (CxiC) objects are proposed in the MAAD structure and the IBCA structure. Then, behavior storing techniques for the multidisciplinary product are explained with the aforementioned concepts. Then, Info-Chunk objects based Information Content (IC) is emphasized. Here, rules for generating the $\mathrm{BiC}$ objects and $\mathrm{CxiC}$ objects in the MAAD structure are defined by using the pseudo-codes. Then, InfoChunkLib API is over viewed. Here, LiC objects of the RFLP structure, BiC and CxiC objects of the MAAD structure are demonstrated. Finally, InfoChunkLib API is imported in the IC to handles the multidisciplinary product model. 


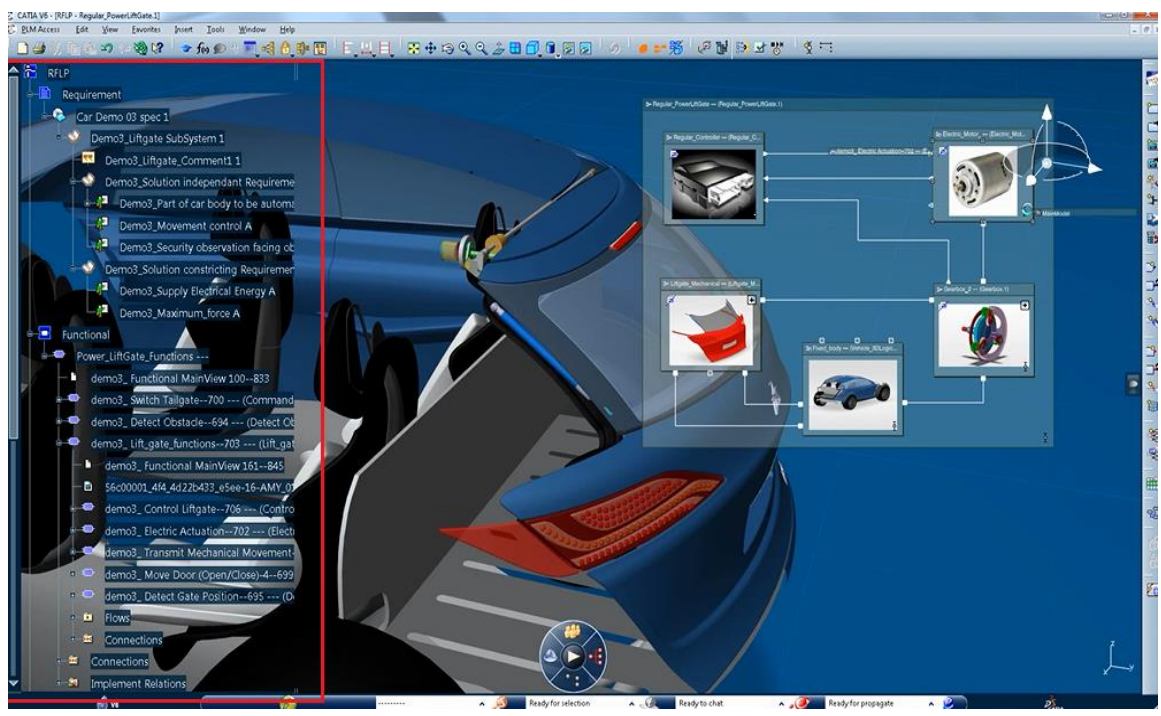

Figure 1

Specification tree of the CATIA V6 RFLP structure

\section{Background}

The Classical Product Model (CPM) [1] is limited to the physical level. The separated integrated mechanical engineering modeling increasingly demands multidisciplinary integration [5]. Modeling of a multidisciplinary product must have a means for the integration of discipline-specific models into a model with a unified structure. It makes the product model virtually executable. Higher abstraction is realized by using of RFLP structure product model [2]. It is compliant with the IEEE 1220 standard. Requirement against the product function to fulfill the requirement, product-wide logical connections, and representations of physically existing objects was organized in the highly contextual RFLP structure.

Human-Computer Interaction (HCI) during the multidisciplinary product modeling is a challenging task. Therefore, Information Content (IC) [1] assists in effective communication between engineers of different disciplines and information-oriented product modeling procedures. Community zones [17] are used in the IC to organize the product model entities and their relationship. Further, behaviors of the modeled entities are evaluated in the information content by the process plane [13]. IC requires MAAD structure to drive the levels of RFLP structure. This structure is used for self-adaptive modeling, where the objectives and requests level, product behaviors level, contexts level, actions 
level, and feature objects are applied in order to connect engineers with RFLP implementations [4]. The MAAD modeling methods and model structures are introduced as a generalized means for the support of higher level abstractionbased generation of RFLP elements. The MAAD modeling was based on the knowledge representation, contextual change propagation, and extended feature definition capabilities for advanced modeling systems [4]. Further, active knowledge in a product model has become organized in the form of Intelligent Property (IP) of the company. Here, IP drives the RFLP level by the IBCA structure which represents active knowledge content [5].

To store the information of a multidisciplinary product, Info-Chunk entity is introduced in the logical level of the RFLP structure [9]. This entity is mapped with the information content to control the structure activities through the MAAD structure. Here, the Layer Info-Chunk (LiC) entity stores the information of the Logical layer and the Component Info-Chunk $(\mathrm{CiC})$ entity stored the information of the logical component. Then, the Info-Chunk entity is defined in the Functional layer of the RFLP structure [19]. Here, the Layer Info-Chunk (LiC) entity stores the information of the main function of the Functional layer of the RFLP structure and Sub-function Info-Chunk (SFiC) entity stores the information of sub-function. Nowadays, OOP concepts are used in system engineering as object-oriented system engineering (OOSE) [3]. OOSE blends system engineering with software engineering.

\section{Info-Chunk as an Object}

In this research work, OOP concepts are used for multidisciplinary product modeling. Encapsulation, inheritance, and polymorphism are the three principles of OOP methodology. This work starts with the entities and their relationship. Info-Chunk [9] [19] is an entity defined in the RFLP structure. However, OOP concepts are not directly applicable to an entity. For InfoChunkLib API, InfoChunk entity must be converted into the Info-Chunk object for communication between IC and RFLP structure. Based on the entity-object conversion process by Ou Y. [10] and Bernhard Thalheim [11]:

- The parameters of an Info-Chunk entity is equivalent to the attribute of an Info-Chunk object

- ER (Entity Relationship) between Info-Chunk is equivalent to the OR (Object Relationship). Here, the method of an Info-Chunk object is derived from the OR as per the requirement of a specific discipline

Then, behavior Info-Chunk (BiC) object and context Info-Chunk (CxiC) object are proposed in the MAAD structure and IBCA structure. According to the proposed concept of Info-Chunk objects: 
- In the RFLP structure, logical layer Info-Chunk (LiCL) object consist of the attributes and methods of the Logical level and functional layer InfoChunk (LiCF) objects consist of the attributes and methods of Functional level

- In the MAAD structure, behavior layer Info-chunk (BiC) object consist of the attributes and methods of Behaviors level and context layer InfoChunk (CxiC) objects consist of attribute and method of Contexts level

- In the IBCA structure, behavior layer Info-chunk (BiC) objects consist of the attributes and methods of Situation defining behaviors $(S B)$ level of Behavior substructures and context layer Info-Chunk (CxiC) objects consist of attribute and method of Product definition Activity Contexts (AC) level, Adaptive Drive Contexts (DC) level, Product Feature Contexts (FC) level of Contexts substructures

\section{Behavior Storing Techniques using Info-Chunk Objects}

Behavior is based on well-defined situations for sets of circumstances. It is represented in the Functional level and Logical level of the RFLP structure. BiC objects and $\mathrm{CxiC}$ objects represent dynamic behavior information. They are stored in the MAAD structure and IBCA structure to communicate with the LiC objects of the RFLP structure. Information Content operates the RFLP structure by the MAAD structure. Also, Intelligent Property (IP) operates the RFLP structure by IBCA structure. The behavior storing techniques are classified as the operation performed by the $\mathrm{BiC}$ objects and $\mathrm{CxiC}$ objects in the MAAD structure and IBCA structure.

\subsection{Info-Chunk Objects-based MAAD Structure}

The Behavior level of the MAAD structure drives the Functional level and Logical level of the RFLP structure. The relationship between the abstraction levels of the MAAD structure is described by using the Unified Modelling Language (UML) diagram as shown in Fig. 2. In the Object-Oriented Programming, a UML diagram is used to define the relationship and model the behavior of the product.

Here, Entities Relationship Modeling (ERM) of the MAAD structure is converted into object relationship modeling (ORM). As per the ORM concept, the relationship between objects is defined by the composition, aggregation, and association. Hence, there is a bi-directional association relationship between the Objectives and Requests level, Behaviors level, Contexts level, and Actions level. 
Inside the Behaviors level, the behavior object has a composite relationship with the situation object, which further has an aggregation relationship with the circumstances object. Also, the behavior object has a bi-directional association with the Adaptive Drive object. In the MAAD structure, a behavior is represented at Behaviors and Contexts level. For behavior representation, communication between the RFLP structure and the MAAD structure is done by using the proposed $\mathrm{BiC}$ objects and $\mathrm{CxiC}$ objects.

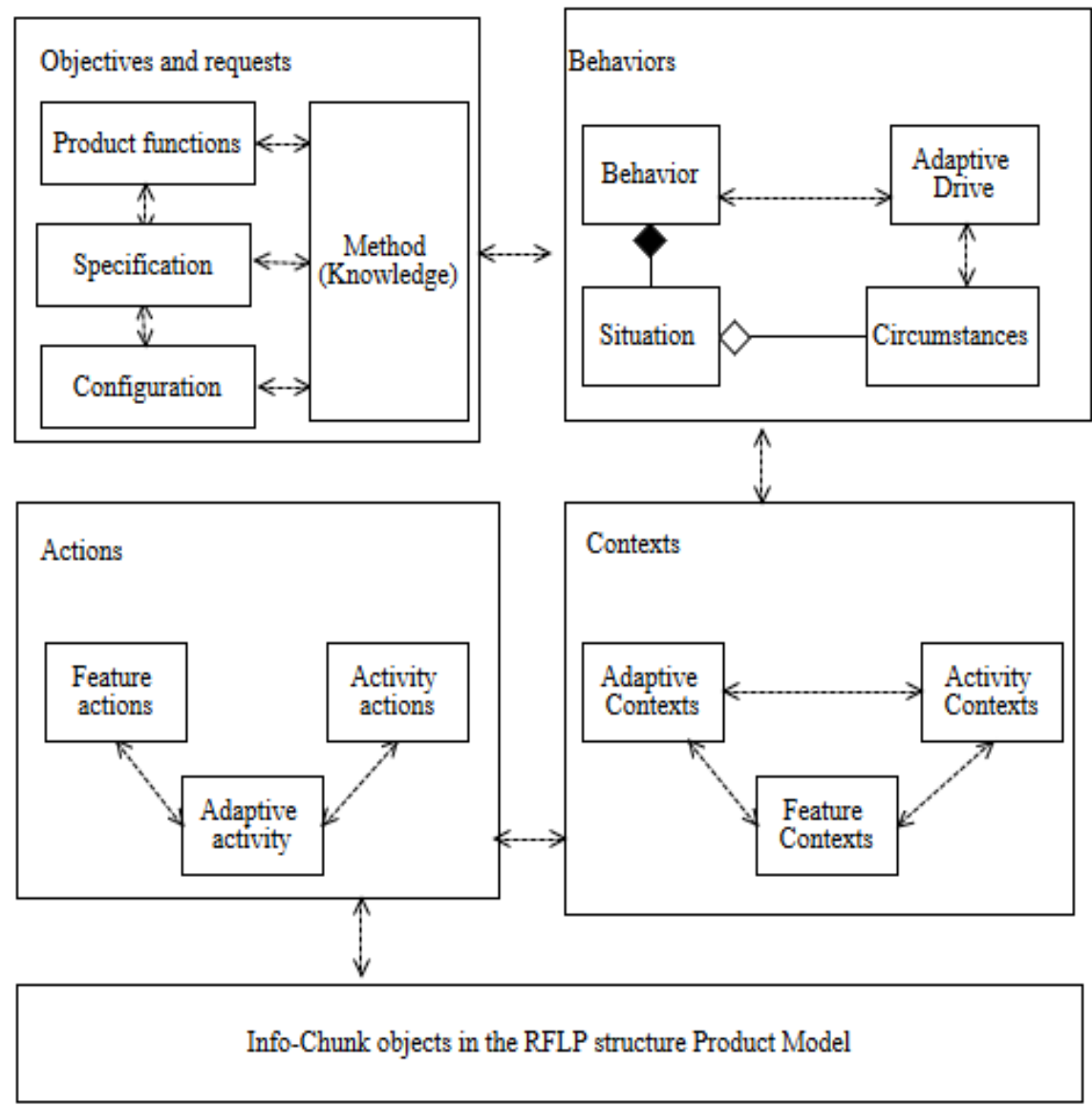

Figure 2

UML representation of MAAD structure with the Info-Chunk based RFLP Structure

The $\mathrm{BiC}$ objects communicate with the LiC objects as shown in Fig. 3, where the main contextual connections of the MAAD structure are organized as follow:

- The solid line is the inside contexts (C) of Behaviors levels for the MAAD structure. It is explained in the paper [18], where the contextual connection of model entities in the MAAD level is defined. 
- The bold line is the driving contexts (D) of Behaviors levels for the MAAD structure. It drives the Functional level and Logical level of the RFLP structure. The dashed lines are the information retrieved by the $\mathrm{BiC}$ objects from the LiC objects of the Functional level and Logical level.

In the case of the Logical layer of RFLP structure, it retrieves the situation attribute of the $\mathrm{LiCL}$ object $\left\{\mathrm{LiCL}_{1}, \mathrm{LiCL} 2, . . \mathrm{LiCLo}\right\}$ and corresponding behavior attribute of their $\mathrm{CiC}$ objects $\left\{\mathrm{CiC} 1, \mathrm{CiC}_{2}, . . \mathrm{CiCn}\right\}$. It is represented inside the oval shape in the diagram. The information retrieved by the driving contexts populates the $\mathrm{BiC}$ objects in the Behaviors level of MAAD structure. Here, $\mathrm{n}$ is the number of $\mathrm{CiC}$ objects in a LiCL object and o is the total number of LiCL objects in the logical layer. The information retrieved is the actual situation, circumstances for the situation and the adaptive drive to drive context definitions.

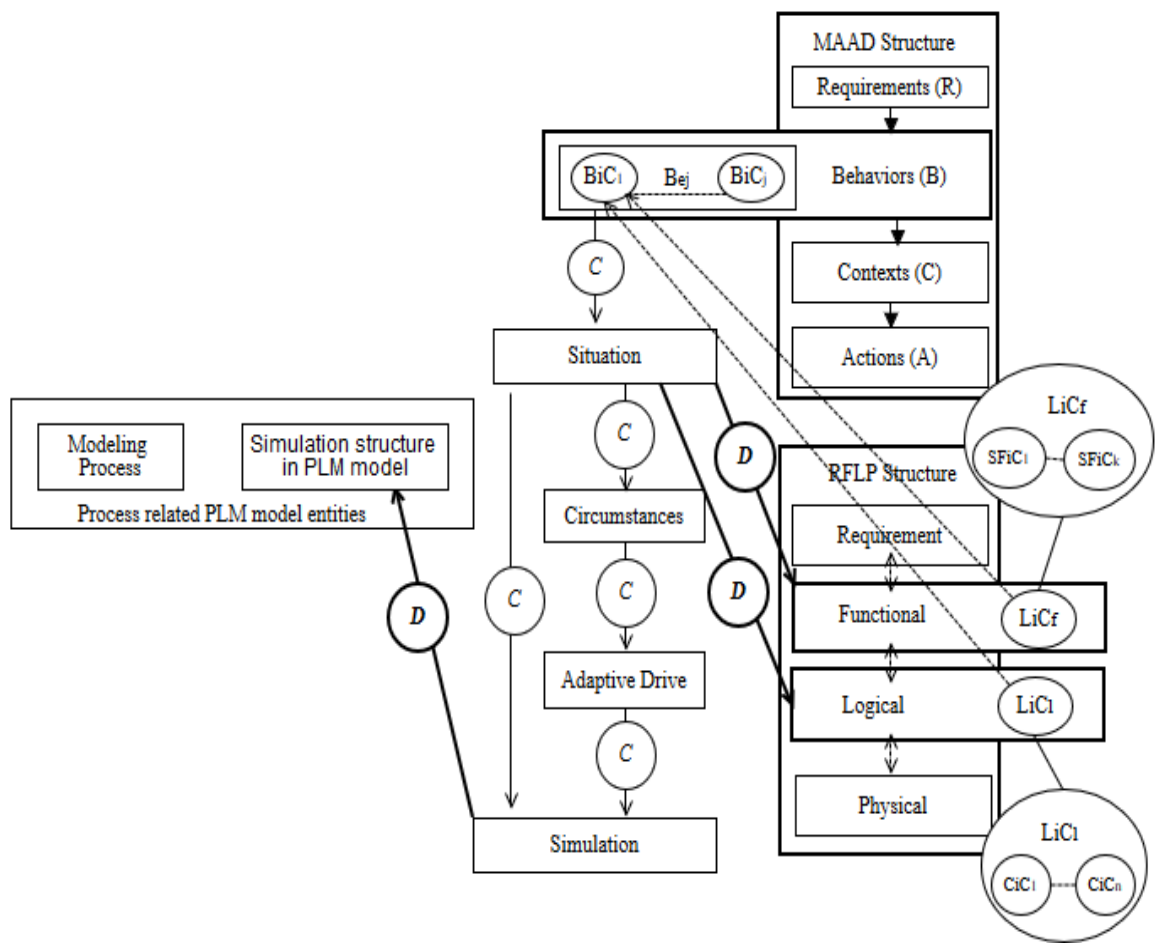

Figure 3

Communication between RFLP and MAAD structure at Behaviors level

In the case of Functional layer of RFLP structure, driving contexts (D) retrieves the requirement class attribute of the $\mathrm{LiCF}$ object $\left\{\mathrm{LiCF}_{1}, \mathrm{LiCF}_{2}, . . \mathrm{LiCF} 1\right\}$ and corresponding elements description attributes of the SFiC objects $\{\mathrm{SFiC} 1, \mathrm{SFiC} 2, .$. $\mathrm{SFiCk}$. It is represented inside the oval shape in the diagram. The information retrieved by the driving contexts populates the $\mathrm{BiC}$ objects in the Behaviors level 
of MAAD structure. Here, $\mathrm{k}$ is the number of SFiC objects in a LiCF object and 1 is the number of LiCF objects in the functional layer of RFLP structure

The retrieved $\mathrm{BiC}$ objects are represented as $\left\{\mathrm{BiC} 1, \mathrm{BiC}_{2}, \ldots \mathrm{BiC}_{\mathrm{j}}\right\}$. Here, $\mathrm{j}$ is the number of $\mathrm{BiC}$ objects in the Behaviors substructure. The $\mathrm{CxiC}$ objects communicate with the LiC objects is shown in Fig. 4, where the main contextual connections of the MAAD structure is organized as follows:

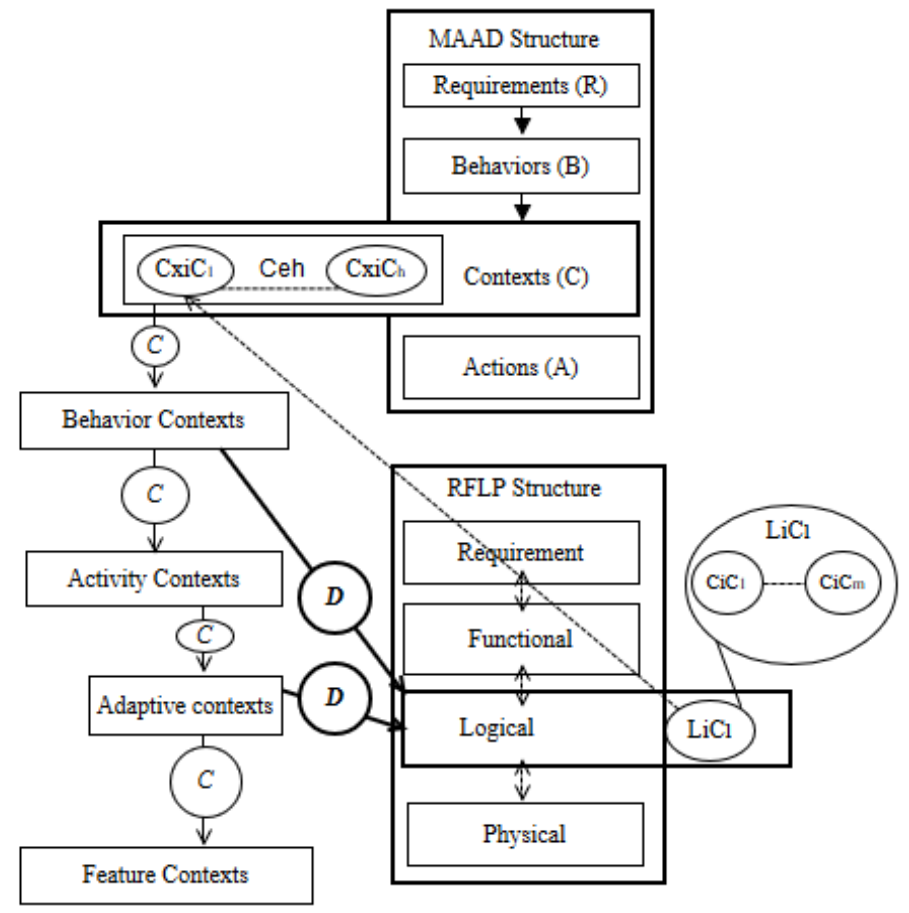

Figure 4

Communication between RFLP and MAAD structure at Contexts level

- The solid line is the inside contexts (C) of Contexts levels for the MAAD structure. It is explained in the paper [18], where the contextual connection of model entities in the MAAD level is defined.

- The bold line is the driving contexts (D) of Behaviors levels for the MAAD structure. It drives the Logical level of the RFLP structure. The dashed line is the information retrieved by the CxiC objects from the $\mathrm{LiC}$ objects of the Logical level.

In the case of the Logical layer of RFLP structure, it retrieves the data model attribute of the $\mathrm{LiCL}$ object $\left\{\mathrm{LiCL}_{1}, \mathrm{LiCL} 2, . . \mathrm{LiCLo}\right\}$ and corresponding data model attribute of $\mathrm{CiC}$ objects $\{\mathrm{CiC} 1, \mathrm{CiC} 2, . . \mathrm{CiCm}\}$. Here, $\mathrm{m}$ is the number of $\mathrm{CiC}$ objects in a LiCL object and o is the total number of LiCL objects in the 
logical layer. The information retrieved is the concept behavior, activity, adaptive and product feature contexts, connection behavior definitions, model definition activities, contexts for an adaptive drive, and context for physical level product and knowledge features. The retrieved $\mathrm{CxiC}$ objects are represented as $\{\mathrm{CxiC} 1$, $\mathrm{CxiC} 2, \ldots \mathrm{CxiCh}\}$, where, $\mathrm{h}$ is the number of $\mathrm{CxiC}$ objects in the Contexts substructure.

\subsection{Info-Chunk Objects-based IBCA Structure}

The driving generation of the RFLP element is done by the Intelligent Property (IP). Human-initiated engineering activities with the company IP by using IBCA structure for the generation of RFLP elements. It leads to the analysis of selfadaptive product lifecycle management (PLM) modeling. The Info-Chunk objects based IBCA structure drives the RFLP structure as shown in Fig. 5. The solid lines are the interaction between the IBCA structure and RFLP structure. The dashed lines are the information retrieved by the $\mathrm{BiC}$ objects and $\mathrm{CxiC}$ objects from the LiC objects of the Functional level and Logical level. On the Behavior (B) level of the IBCA structure, situations defining behaviors (SB) substructure are configured to define behaviors by a set of $\mathrm{BiC}$ objects.

- In the Logical level of the RFLP structure, the situation attribute \& behavior attribute of the LiCL object $\left\{\mathrm{LiCL}_{1}, \mathrm{LiCL} 2, \ldots \mathrm{LiCLd}\right\}$ and the corresponding behavior attribute of the $\mathrm{CiC}$ objects $\{\mathrm{CiC} 1, \mathrm{CiC} 2, \ldots$ $\mathrm{CiCa}$ are stored in the $\mathrm{BiC}$ objects of the $\mathrm{SB}$ element. Here, a is the number of $\mathrm{CiC}$ objects in a $\mathrm{LiCL}$ object and $\mathrm{d}$ is the total number of LiCL objects.

- In the Functional level of the RFLP structure, requirement attribute of the $\mathrm{LiC}$ object $\{\mathrm{LiC} 1, \mathrm{LiC} 2, \ldots \mathrm{LiCc}\}$ and corresponding elements description attributes of the SFiC objects $\{\mathrm{SFiC} 1, \mathrm{SFiC} 2, . . \mathrm{SFiCb}\}$ are stored in the $\mathrm{BiC}$ objects of the SB element. Here, $\mathrm{b}$ is the number of SFiC objects in a $\mathrm{LiC}$ object and $\mathrm{c}$ is the number of $\mathrm{LiC}$ objects in the functional level.

The stored information in the $\mathrm{BiC}$ objects is behavior definition (IEBD) and the related situation (IEBT) [12]. The total $\mathrm{BiC}$ objects obtained from the LiC objects of the functional and logical layer is represented as $\left\{\mathrm{BiC}_{1}, \mathrm{BiC} 2, \ldots \mathrm{BiCn}\right\}$. Here, $\mathrm{j}$ is the number of $\mathrm{BiC}$ objects in the SB element. On the Contexts (C) level of the IBCA structure, product definition activity contexts (AC) level, adaptive drive contexts (DC) level, and product feature contexts $(F C)$ level are configured to define behaviors by a set of CxiC objects. In the logical level of the RFLP structure, the data model attributes of $\mathrm{LiC}$ objects $\{\mathrm{LiC} 1, \mathrm{LiC} 2, \ldots \mathrm{LiCd}\} \& \mathrm{CiC}$ objects $\left\{\mathrm{CiC}_{1}, \mathrm{CiC} 2, . . \mathrm{CiCa}\right\}$ are stored by the $\mathrm{CxiC}$ objects of $\mathrm{AC}, \mathrm{DC}$ and $\mathrm{FC}$ elements. Here, a is the number of $\mathrm{CiC}$ objects in a $\mathrm{LiC}$ object and $\mathrm{d}$ is the total number of $\mathrm{LiC}$ objects. The stored information in the CxiC objects is the product behavior (IECB). The total CxiC objects obtained from the LiC objects of logical 
layer is represented as $\{\mathrm{CxiC} 1, \mathrm{CxiC} 2, . . \mathrm{CxiC}\},\{\mathrm{CxiC} 1, \mathrm{CxiC} 2, . . \mathrm{CxiCy}\}$, $\{\mathrm{CxiC} 1, \mathrm{CxiC} 2, . . \mathrm{CxiC}\}$. Here, $\mathrm{x}, \mathrm{y}, \mathrm{z}$ are the number of $\mathrm{CxiC}$ objects stored in the $\mathrm{AC}, \mathrm{DC}$ and FC elements.

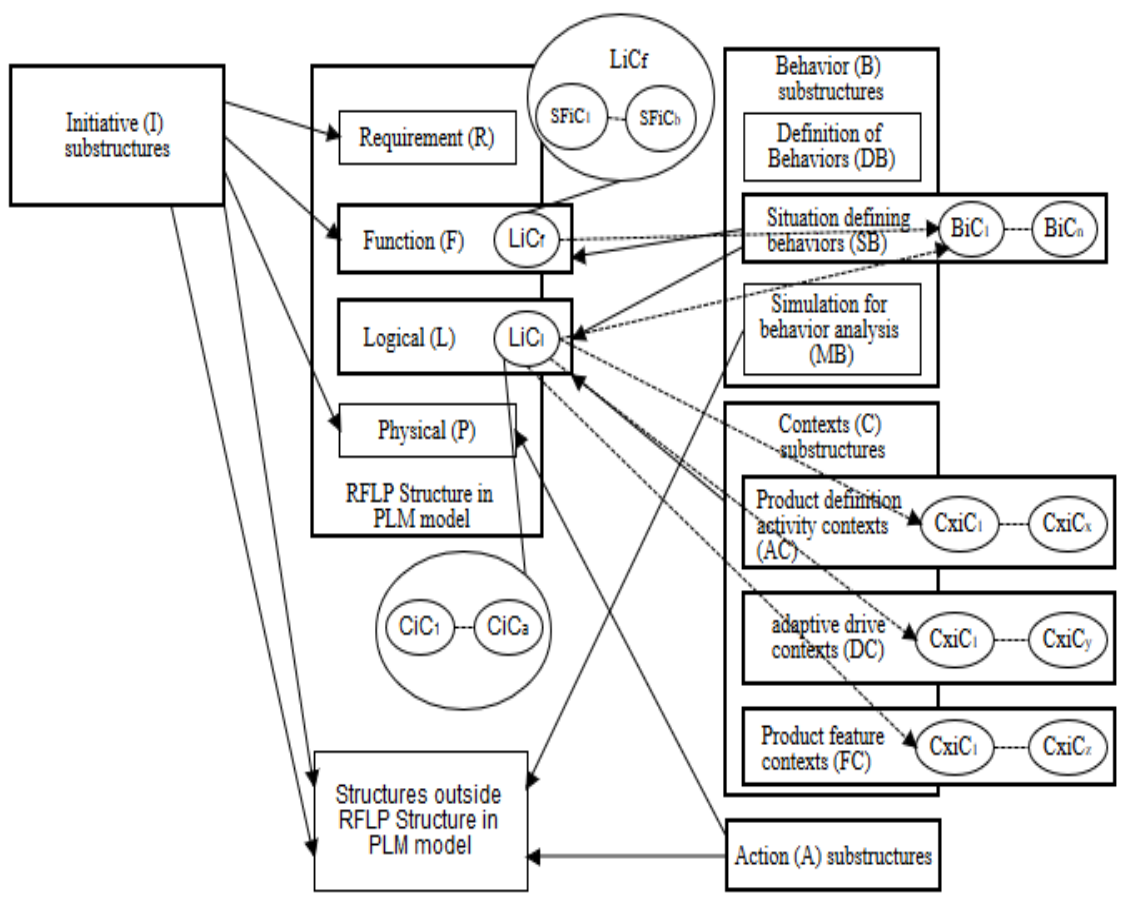

Figure 5

Communication between RFLP and IBCA structure at Behavior and Contexts substructure

\section{Info-Chunk Objects-based Information Content}

Behavior models with intelligent content involve specifications and knowledge for the design processes. The most appropriate forms of knowledge are formulas, rules, and checks. In the following sections, this work focuses on Info-Chunk object activities in the information content (IC). Here, the MAAD structure is the driving factor for representing the behavior of the RFLP structure.

\subsection{Rules for the Generation of Info-Chunk Objects}

Rules are the set of instructions that can be executed for generating and storing the Info-Chunk objects in the MAAD and IBCA structure. Rules are defined by using pseudo-codes. 
- In the case of the MAAD structure, the behavior objects $\{\mathrm{BiC} 1, \mathrm{BiC} 2, \ldots$ $\left.\mathrm{BiC}_{\mathrm{j}}\right\}$ are stored in the behaviors level and the context objects $\{\mathrm{CxiC} 1$, $\mathrm{CxiC} 2, \ldots \mathrm{CxiCh}\}$ are stored in the contexts level. The Process plane of IC [13] can elaborate on the $\mathrm{BiC}$ and $\mathrm{CxiC}$ objects for the behavior representation of the multidisciplinary product. After the analysis process, the analyzed objects are stored with the nomenclature of $\mathrm{BiCab}$. If a human wants to evaluate the context of one analyzed object on the other analyzed object, the context object undergoes the effect process. The resultant objects are stored as CxiCec. Further, If a human wants to optimize the contextual object, it is stored as BiCob after the optimization process. It is also possible to optimize the behavior of an object without analysis. Information content (IC) retrieve and store required objects at the Engineering objectives level to drive the behavior of RFLP structure.

- In the case of the IBCA structure, the behavior objects $\{\mathrm{BiC} 1, \mathrm{BiC} 2, .$. $\mathrm{BiCn}\}$ are stored in the behavior substructure and the context objects $\{\mathrm{CxiC} 1, \mathrm{CxiC} 2, . . \mathrm{CxiC} x\},\{\mathrm{CxiC} 1, \mathrm{CxiC} 2, . . \mathrm{CxiCy}\},\{\mathrm{CxiC} 1, \mathrm{CxiC} 2, .$. $\mathrm{CxiC} z$ are stored in the contexts substructures. IP could retrieve and store these objects to drive the behavior of the RFLP structure. The IP level and process plane of IP are not defined yet. The behavior representation for IP is the topic of future work.

\section{Pseudo Codes for BiC \& CxiC objects}

- BEGIN LOOP

- Initialize a Process

- IF 'Process' is 'Analysis'

- BEGIN LOOP

- Store 'BiCab' in 'Behaviors level' where $1 \leq \mathrm{ab} \leq \mathrm{j}$

○ IF 'Process' is 'Effect'

- BEGIN LOOP

- Store 'CxiCac' in 'Contexts level' where $1 \leq \mathrm{ac}$ $\leq \mathrm{h}$

- IF 'Process' is 'Optimization'

* BEGIN LOOP

* Store 'BiCob' in 'Behaviors level' where $1 \leq$ $\mathrm{ob} \leq \mathrm{j}$

* END LOOP

- END LOOP

○ END LOOP

- IF 'Process' is 'Optimization' 


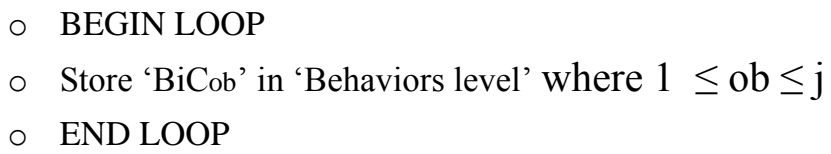

\section{Overview of the InfoChunkLib API}

The InfoChunkLib API is coded in the JavaFX application as shown in Fig. 6. It consists of two Java packages. The informationcontent Package consists of all the classes related to the Information Content (IC) like MAADStructure class, BiC class, $\mathrm{CxiC}$ class, and CommunityZone class. The rflp Package consists of all the classes related to the RFLP structure like $L i C L$ class, $L i C F$ class, $C i C$ class, and SFiC class.

\subsection{Demonstration of Info-Chunk Objects in the RFLP Structure}

To explain the proposed concepts in the system behavior, let us consider a car as an example. According to the community concepts [17], a car system is the combination of various communities where the Electrical supply system is one of the community. It consists of components like battery, starter, alternator, heater, fan, distributor, etc. Here, battery and alternator components are used for the InfoChunk objects concept explanation. Then, the following scenario is considered as an example: The oil consumption of car depends on the engine behavior that must be modeled according to the situation such as the experience of the driver with the sets of circumstances like path traveled by car, the condition of the car, surrounding environment, etc.

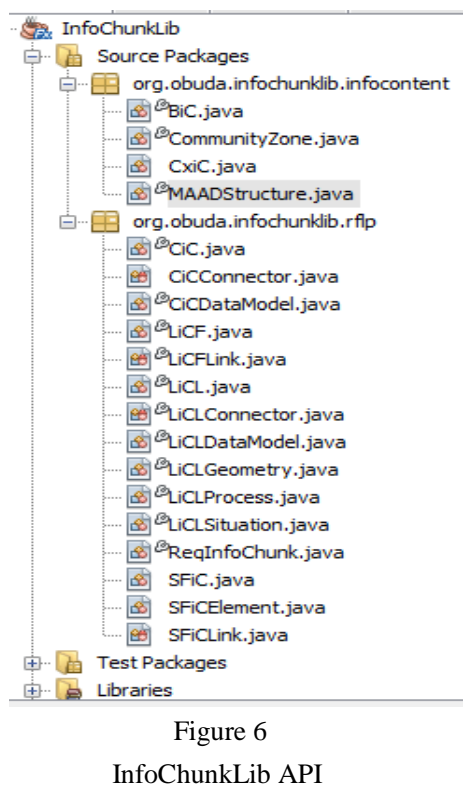

Here, the dynamic behavior of the engine strongly is influenced by the situation and weakly influenced by the circumstances. The parameters of the LiCL objects and $\mathrm{CiC}$ objects are described in the paper [9]. Also, the parameters of $\mathrm{LiCF}$ objects and SFiC objects are described in the paper [19]. The descriptions of LiCF 
class and LiCL class are shown in the code below. The $L i C F$ class is written to show the layer Info-Chunk object of the functional layer in the RFLP structure. Here, the array of SFiC objects, ReqInfoChunk object, LiCFLink object and String parameters are used as an argument in the constructor. The constructor arguments of the ReqInfoChunk object are populated from the Requirement layer of the RFLP structure. The ReqInfoChunk class is written to show the customer requirements in the requirement layer of the RFLP structure. The SFiC class is written to show the sub-functional Info-Chunk object in the functional layer of the RFLP structure. The LiCFLink is the enumeration class to store connector information. The concepts of constructor overloading are used so that LiCF can accept various sets of the argument depends on the initialization of the object.

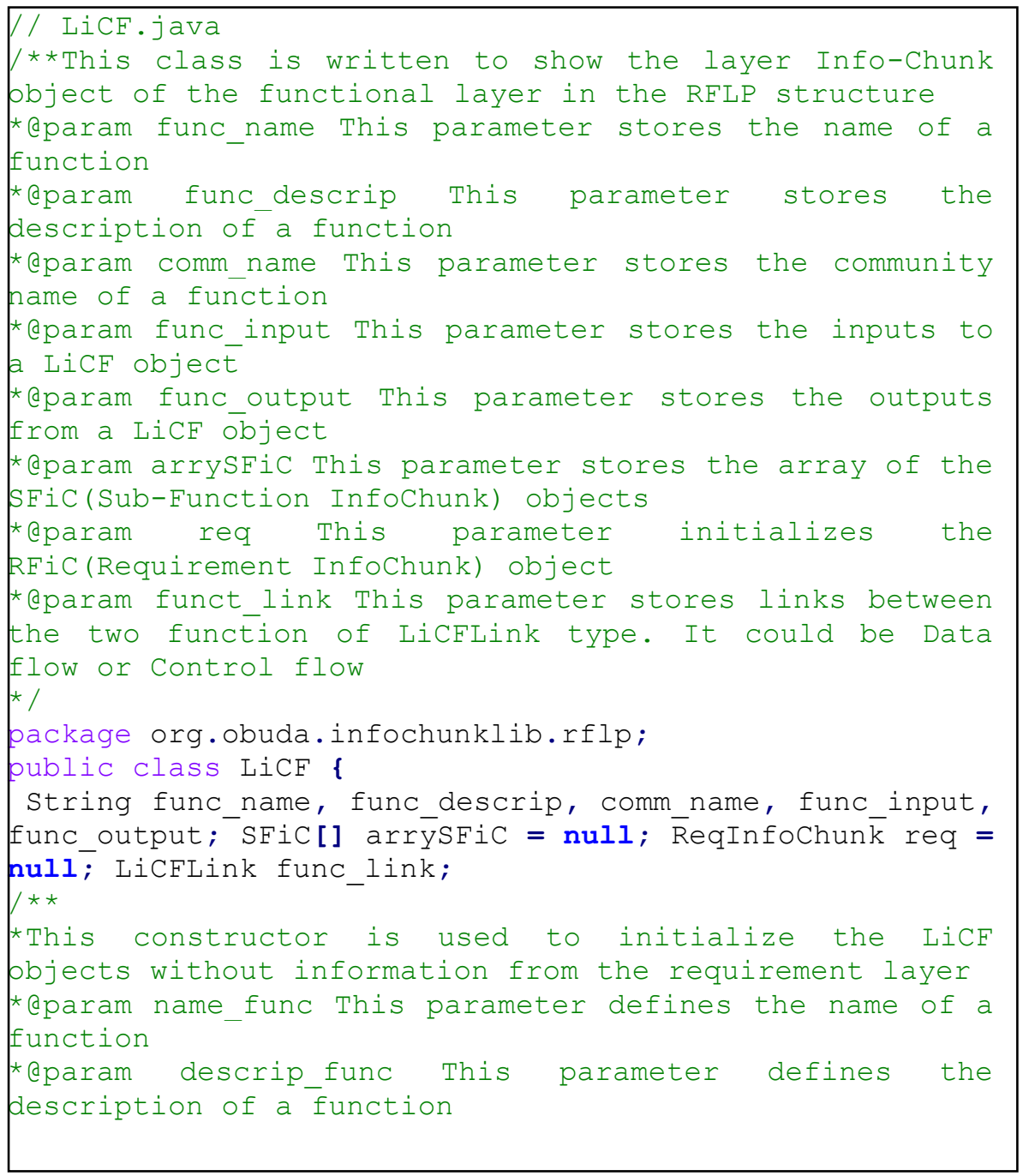




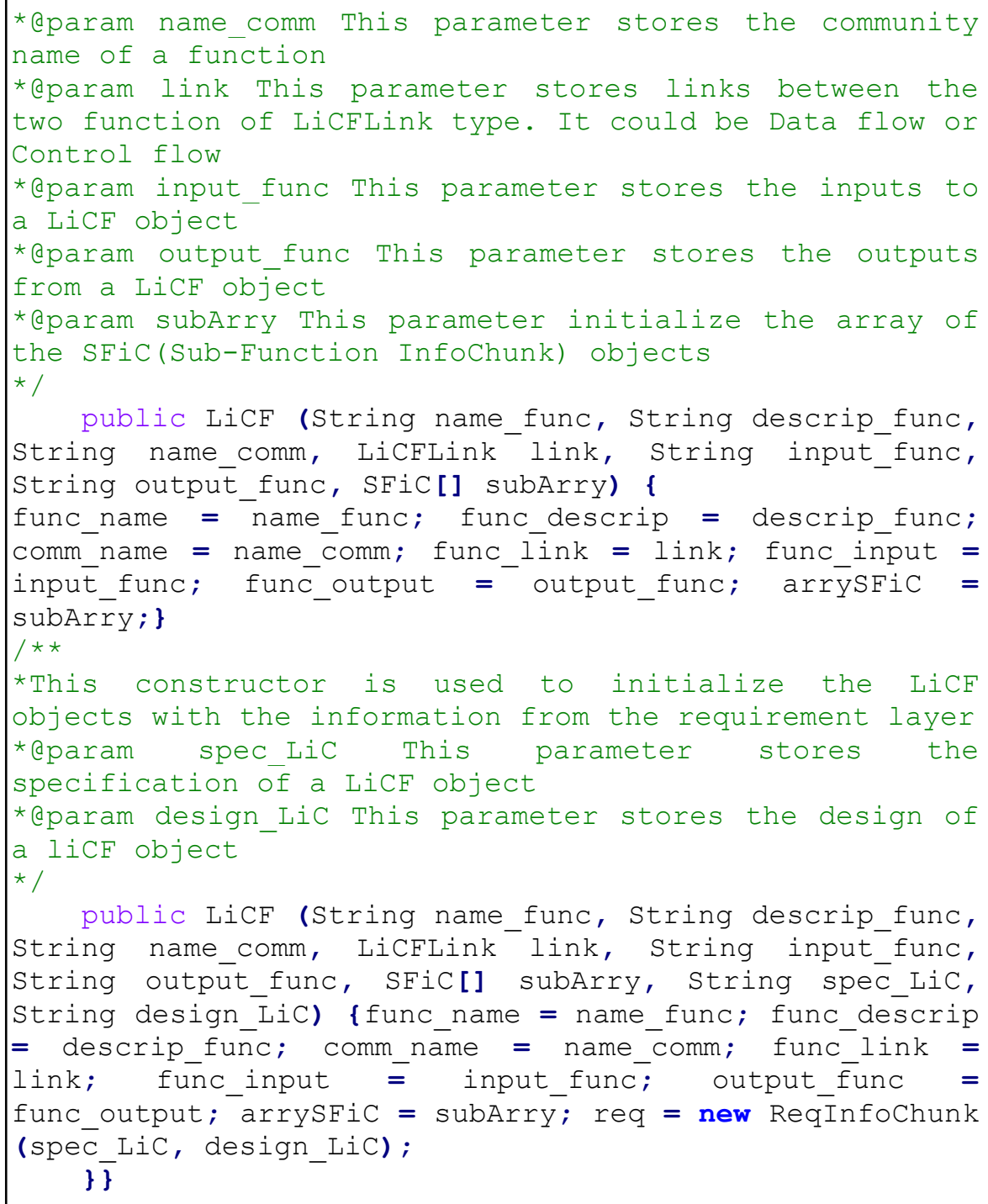

Further, the LiCL class is written to show the layer Info-Chunk object of the logical layer in the RFLP structure. Here, the array of CiC objects, LiCLGeometry object, LiCLSituation object, LiCLProcess object, LiCLDataModel object, LiCF object, LiCLConnector object, integer parameter, boolean parameter, string parameters, and the array of string parameters are used as an argument in the constructor. The constructor arguments of a LiCF object are populated from the Functional layer of the RFLP structure. The concepts of constructor overloading are used so that LiCL can accept various sets of the argument depends on the initialization of the object. The $\mathrm{CiC}$ class is written to show the component InfoChunk object in the logical layer of the RFLP structure. The LiCLGeometry class 
is written to show the geometry of the multidisciplinary product. Here, it could be possible for data retrieval of the product model and part model's STEP files in the LiCL class. In that case, LiCLGeometry constructor's arguments part_info and assembly_info are converted from string types into the STEP file format. Here, JSDAI API could be the possible approach to read and write the STEP file format. Then, LiCLGeometry object, affect zone and array of circumstances are used as a constructor argument for the LiCLSituation object. The LiCLSituation class is written to show the situation with a set of circumstances applicable to a LiCL object. The LiCLProcess class is written to show the process plane of the IC. It accepts String and Boolean values of processes as a constructor argument. The string values store the name of the processes whereas Boolean value stores the status of a process. The LiCLConnector is the enumeration class to store connector information. The get method returns the value of objects required to the main application. It is used in the next subsection.

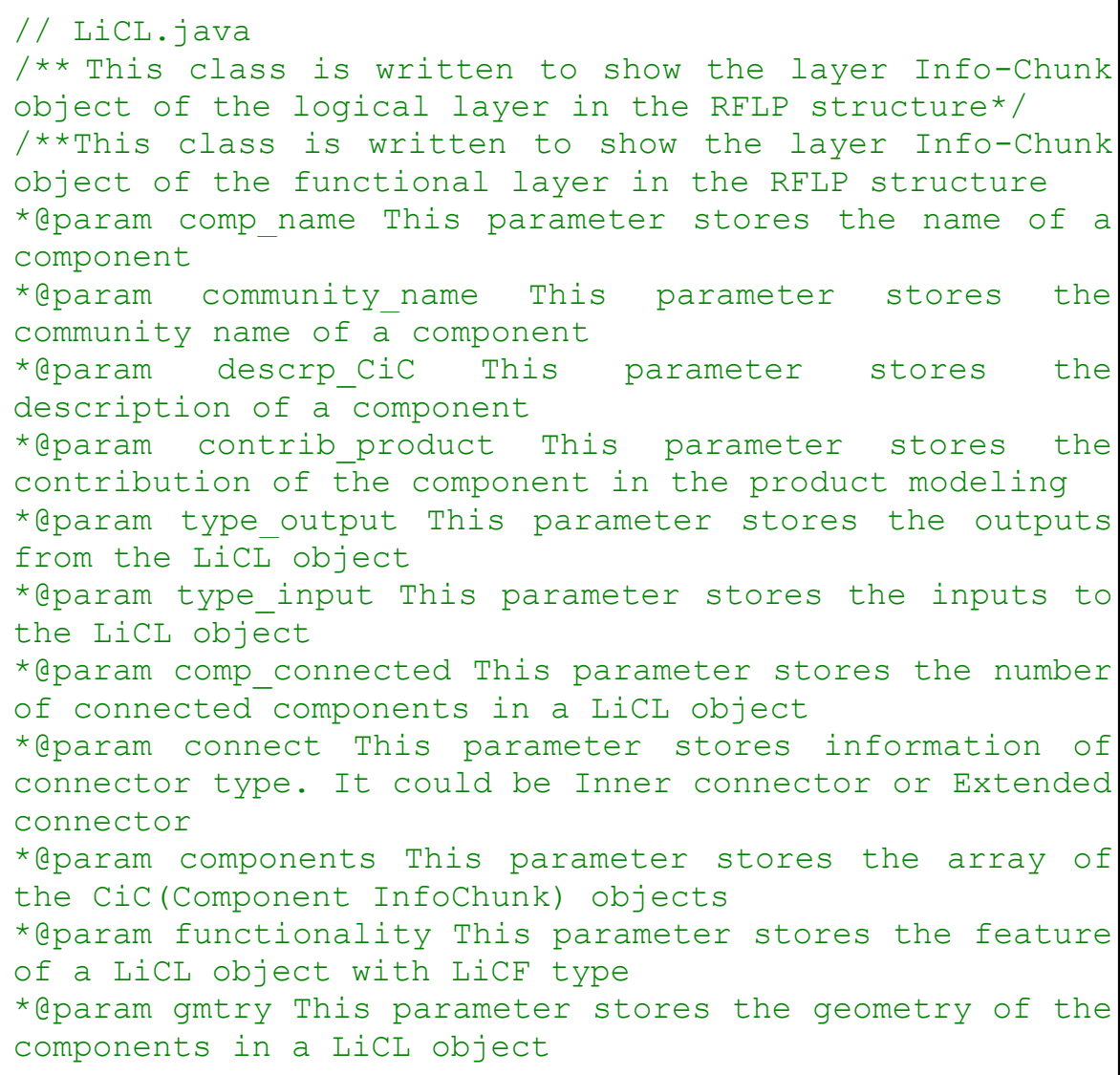


* Aparam situation This parameter stores the information of influenced components and geometry of the components in a LiCL object at the given situation

* Aparam process This parameter stores the process involved in a LiCL object

* eparam data model This parameter stores the detail description of a LICL object in the context of the physical object such as process, geometry, and situation

*/

package org.obuda.infochunklib.rflp;

public class LiCL \{

private string comp_name, community name, descrp_cic, contrib_product, type_input, type_output; - int comp connected; LicLConnēctor connect; Cic[] components $=$ null $;$ LiCF functionality = null; LiCLGeometry gmtry = null; LiCLSituation situation = null; LiCLProcess process $=$ null; LiCLDataModel data model = null;

$1 * *$

*This constructor is used to initialize the LiCL object without the information to the physical layer

* ¿param name_comp This parameter stores the name of a component

* Aparam name_community This parameter stores the community name of a component

* Aparam connected comp This parameter stores the number of connected components in a LiCL object

* Aparam product contib This parameter stores the contribution of the component in the product modeling

* Aparam input_type This parameter stores the inputs to the LiCL object

* Aparam output type This parameter stores the outputs from the LiCL object

* eparam affect_zone This parameter stores the influenced components during the analysis in a LiCL object

* eparam part_info This parameter stores part information in the geometry of a LiCL object

* ¿param assembly_info This parameter stores assembly information in the geometry of a LiCL object

* @param form_features This parameter stores form feature information in the geometry of a LiCL object

* eparam circum This parameter stores the array of the circumstance of a situation in a LiCL object

* eparam arrycic This parameter stores the array of the CiC(Component Infochunk) objects 
* Aparam function This parameter stores the feature of a LiCL object with LiCF type

* /

public LicL(String name_comp, String name_community, int connected_comp, string product_contib, String input_type, string output_type, String affect_zone, Strīng part info, String assembly_info, String form_features, string[] circum, CiC[] arryCiC, LiCF function) \{

comp_name $=$ name_comp; comp_connected $=$ connected_comp; community_name = name_community; contrib_prodūct = product_contib; type_input = input_type; type_output = output_type; functionality = function; ${ }_{\text {- connect }}$ =connection; components = arrycic; gmtry = new LiCLGeometry(part_info, assembly_info, form_features); situation = new LicLSituation(affect_zonē, circum, gmtry);

/**

*This constructor is used to initialize the LiCL object with the information to the physical layer

* eparam process analysis This parameter stores the status of the anālysis process in a LiCL object

* Aparam process_effect This parameter stores the status of the effect/contextual process in a LiCL object

* eparam process optimization This parameter stores the status of the optimization process in a LiCL object

* @param value analysis This parameter stores the array of analysis process values in a LiCL object

* Aparam value_effect This parameter stores the array of contextual process values in a LiCL object

* eparam value optimization This parameter stores the array of optimization process values in a LiCL object

* Aparam connection This parameter stores information of connector type. It could be Inner connector or Extended connector

* @param contexual_PO This parameter stores knowledge of contextual Physical object/s in a LicL object

* @param connected_PO This parameter stores knowledge of connected Physical object/s in a LicL object */

$$
\text { public LicL(String name_comp, String }
$$
name_community, int connected_comp, string product_contib, string input_type, string output_type, String affect_zone, String part_info, S̄tring assembly_info, String form_features, String[] circum, Boolean process_analysis, Boolean process_effect, 


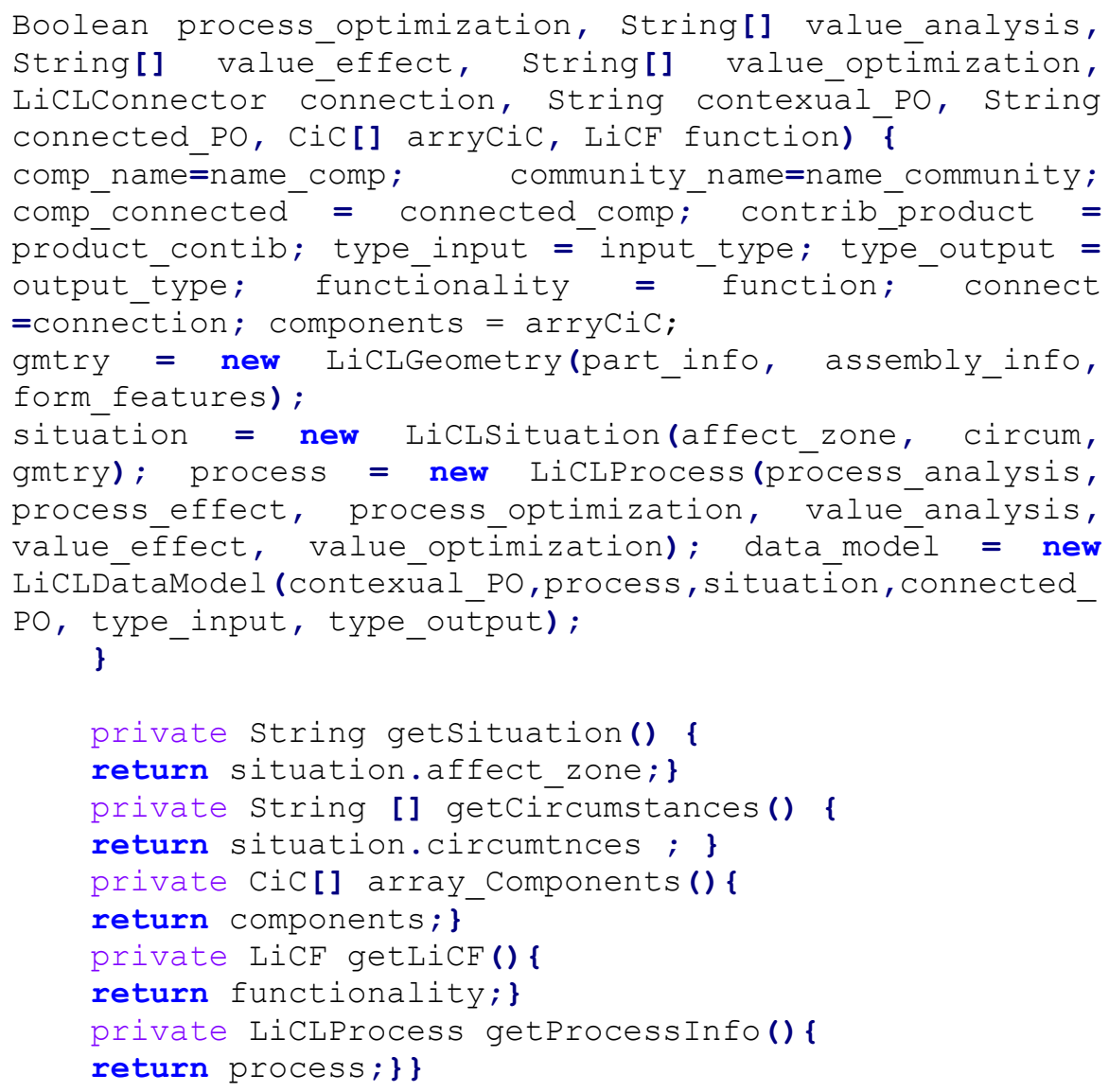

\subsection{Demonstration of Info-Chunk Objects in MAAD Structure}

The $B i C$ and $C x i C$ class are the application classes for the behavior representation of the multidisciplinary product model as shown in the code below. The output is the graph between the components of various disciplines. It is the outcome of the process plane of the IC. The $B i C$ class accepts the LiCL and LiCF objects as a constructor argument. Using the LiCL object, the LiCLProcess object can check the status of the analysis and optimization process. If the value is true, then it can generate the graph related to the process. The outcome of the Analysis process is shown in Fig. 7. The graph explains the displacement of the battery, starter and attenuator components w.r.t to time after the Thermal Analysis process. The outcome of the optimization process is shown in Fig. 8. The graph explains the voltage required w.r.t time for the optimized battery response. 


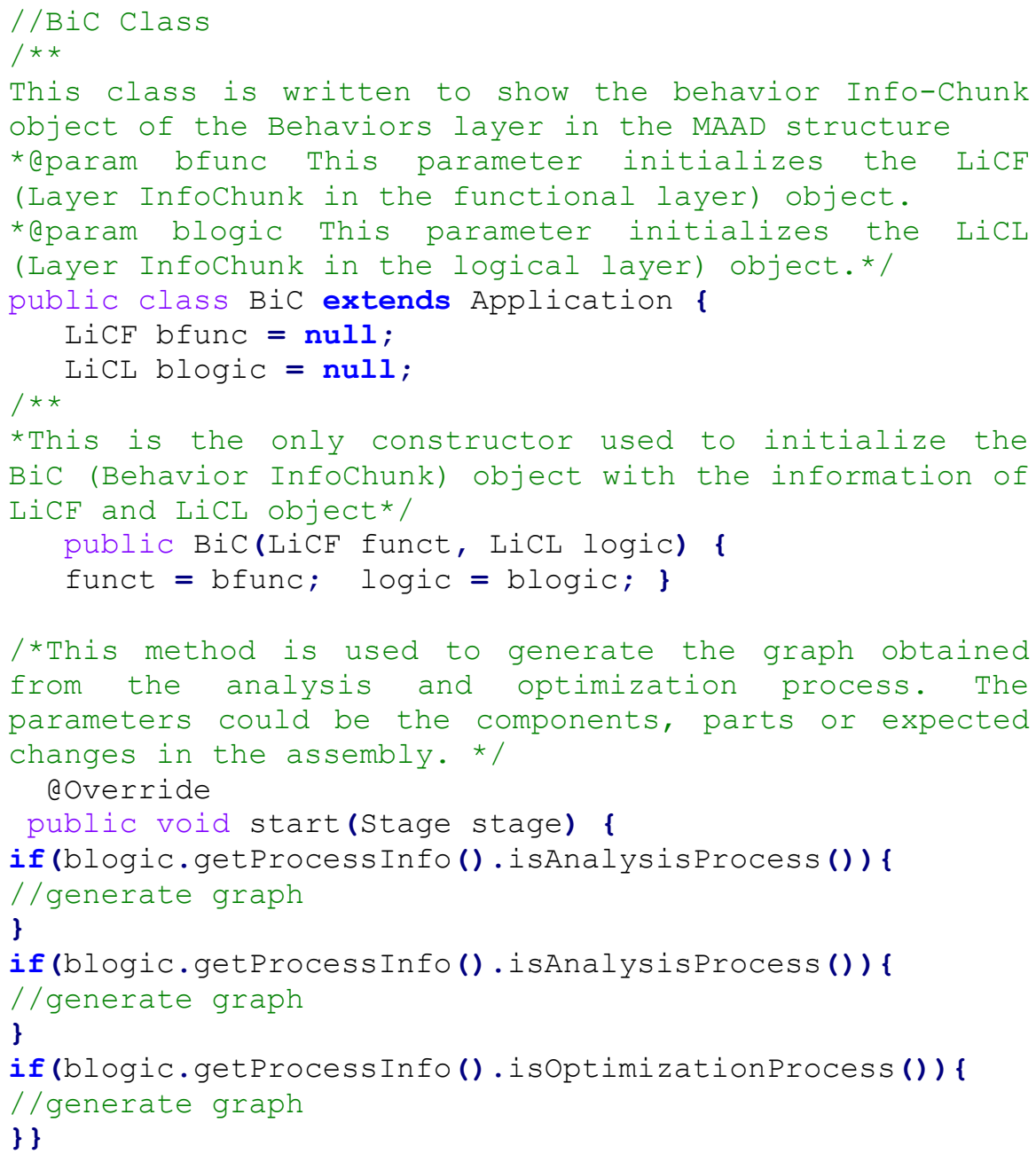

The CxiC class accepts LiCL object as a constructor argument. Using LiCL object, the LiCLProcess object can check the status of the effect (contextual) process. If the value is true, then it can generate the graph based on the contextual relationship between engineering objects. The outcome of Effect process is shown in Fig. 9 where contextual relation between attenuator and battery is explained by varying the battery output current with the attenuator speed. Here, XYChart class is used for generating the Line Chart graph. 
//CxiC Class

$/ * *$

This class is written to show the Contexts Info-chunk object of the Behaviors layer in the MAAD structure

* eparam blogic This parameter initialize the LiCL (Layer Infochunk in the logical layer) object */

public class CxiC extends Application \{

LiCL blogic = null;

$1 * *$

*This is the only constructor used to initialize the Cxic (Contextual Infochunk) object with the LiCL object* /

public CxiC(LiCL $\operatorname{logic})$ \{logic $=$ blogic;

/**This method is used to generate the graph obtained from the contextual process. The parameters could be the components, parts or expected changes in the assembly * * /

@override

public void start (Stage stage) \{

if (blogic.getProcessInfo(). isEffectProcess ()) \{

//generate graph

\} \}

The MAADStructure class is the main method class that launches the application by calling the objects of $\mathrm{BiC}$ and $\mathrm{CxiC}$ objects.

//MAADStructure Class

/** This class is written to show the main application

of the InfochunkLib API. */

public class MAADStructure \{

/** This method is used to start the main application by calling the BiC and CxiC objects and generate the graphs*/

public static void main(String args[]) throws Exception \{

Application.launch(BiC.class, args);

new Thread () \{

Application.launch(CxiC.class, args);

\} \} \} 


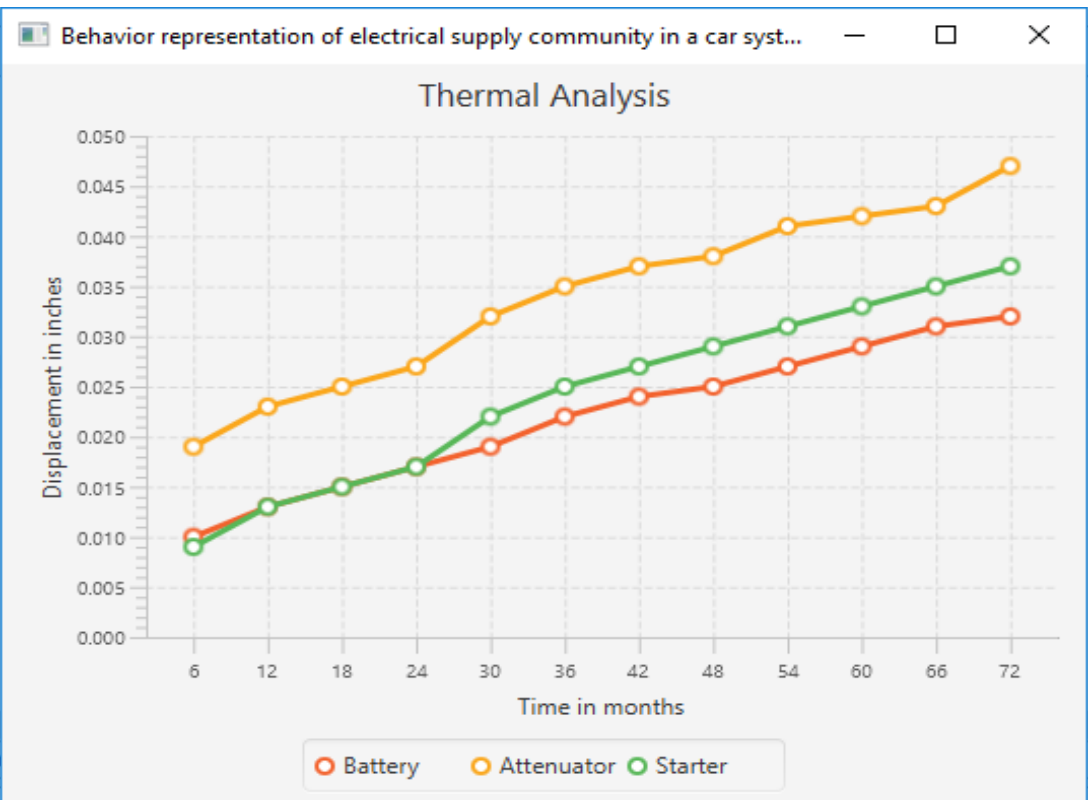

Figure 7

The graph of components after thermal analysis

11 Behavior representation of electrical supply community in a car system $\quad-\quad \square \quad \square$ optimized Battery response to load removal

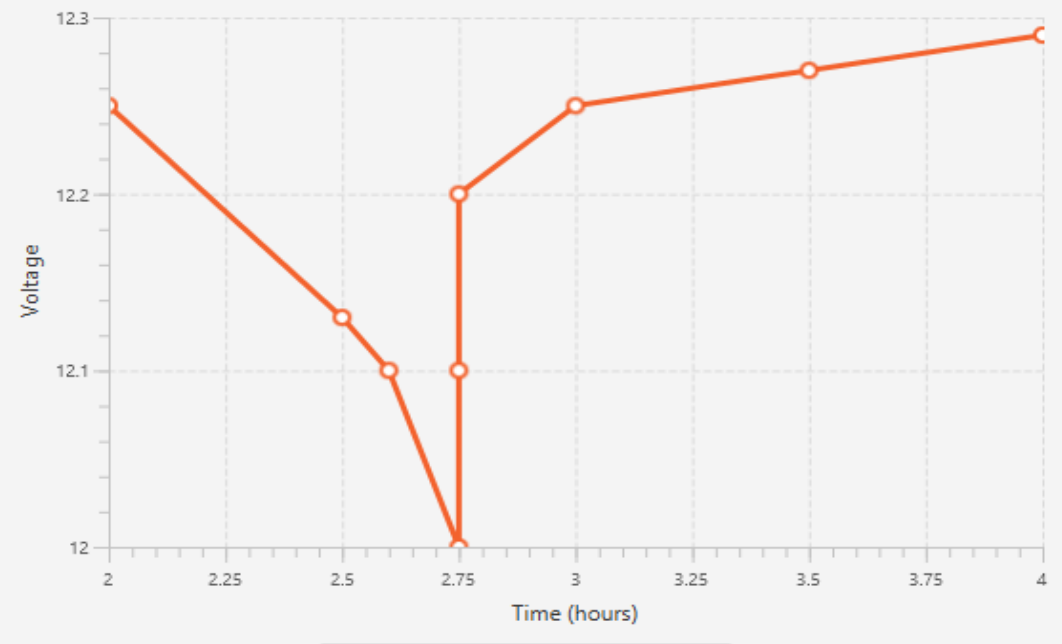

Voltage supplied to car components

Figure 8

The graph of the battery component after the optimization process 


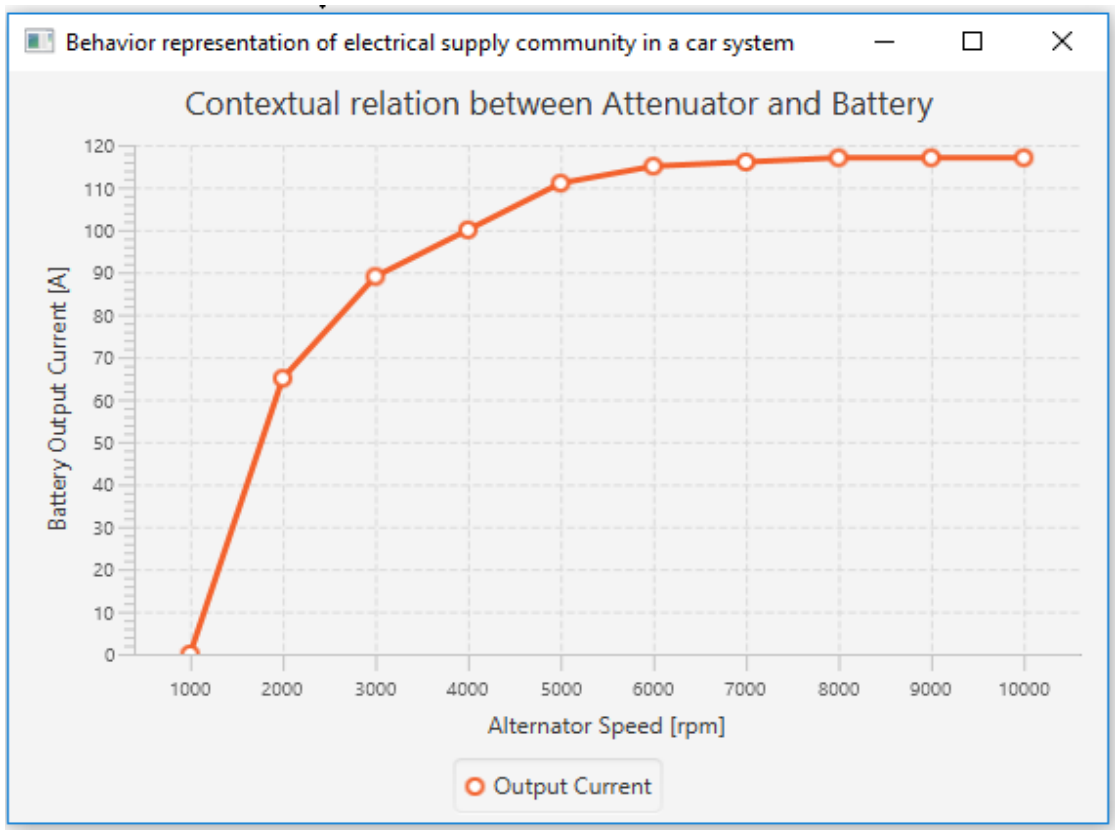

Figure 9

The graph between battery and attenuator component after effect/contextual process

\subsection{Implementation of InfoChunkLib API in the Information Content}

InformationContent class is the application which imports the InfoChunkLib API and handles the multidisciplinary product model. It could be a Java application or Web application. The output is stored in the database. As shown in the code below, SFiC objects and $\mathrm{CiC}$ objects are initialized first. Then, LiCF objects are initialized from SFiC objects and LiCL objects are initialized from CiC objects and LiCF objects. Then, BiC objects are initialized by LiCF objects and LiCL objects. CxiC objects are initialized by LiCL objects. Finally, the MAADStructure class is called by InformationContent arguments and graphs are generated for the behavior representation of multidisciplinary product model.

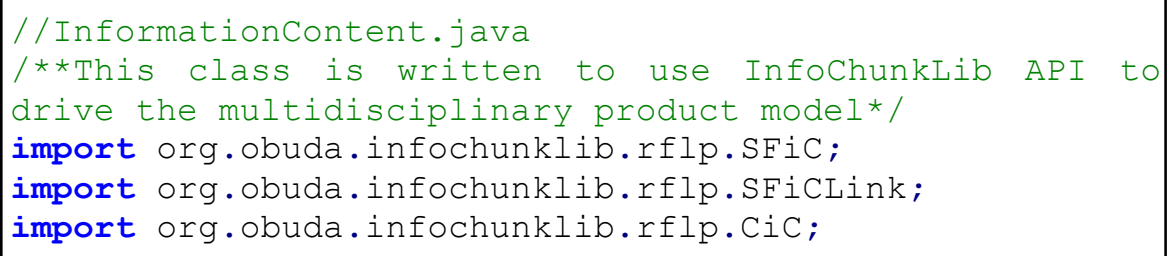




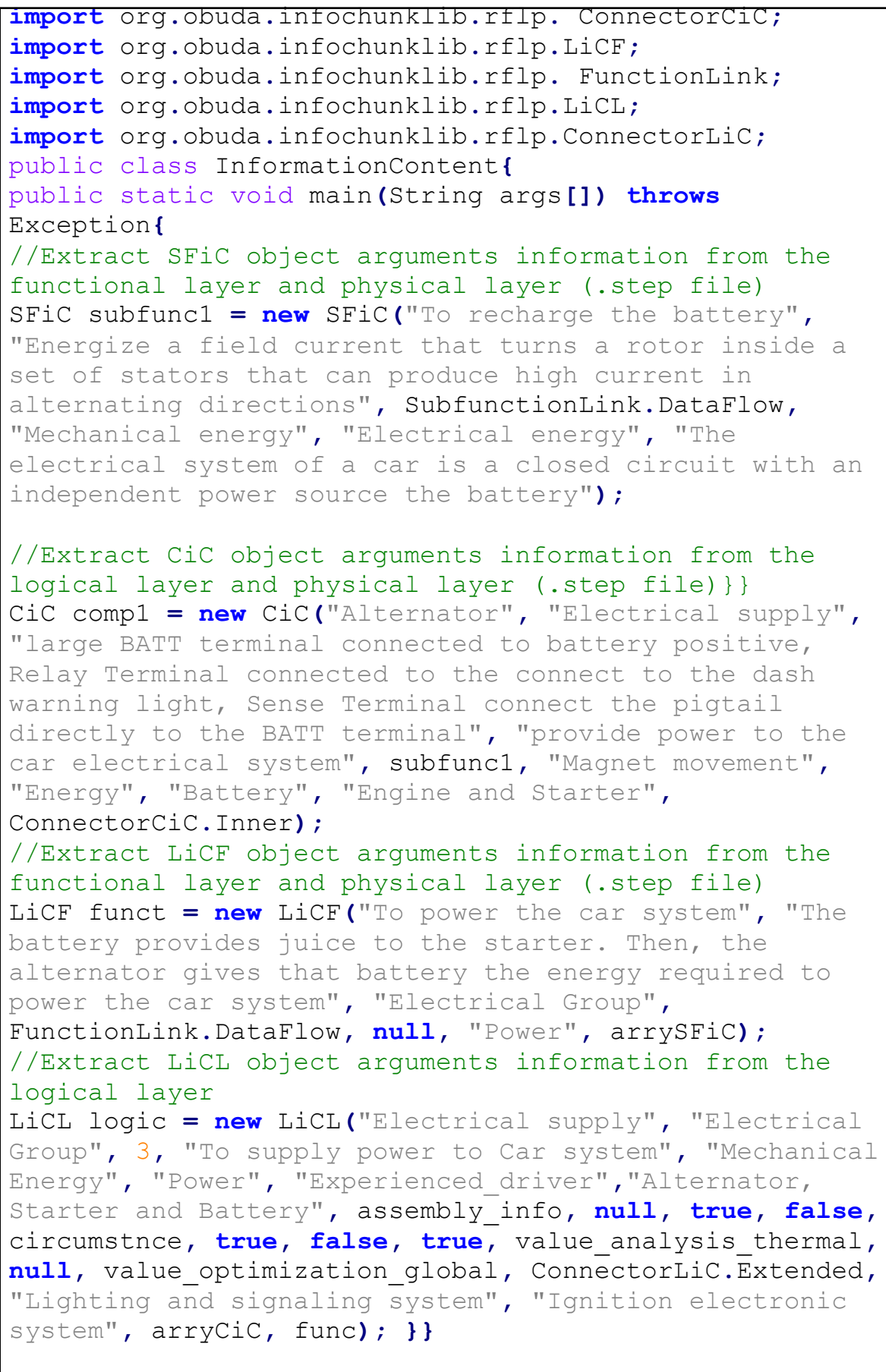




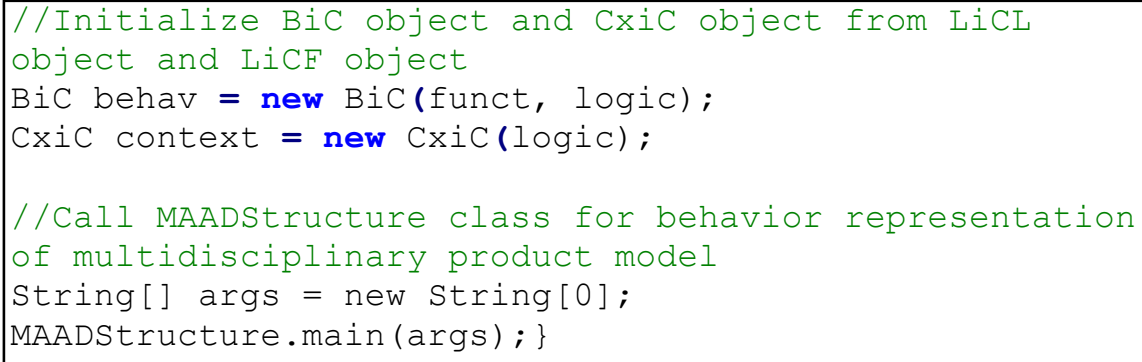

\subsection{Testing Phase of the Info-Chunk Objects}

It is necessary to check the stored information in the Info-Chunk objects. In the OOP based language like Java, JUnit testing is a popular tool to check the behavior of an object. The behavior of a multidisciplinary product can be tested by varying the attributes and methods of the $\mathrm{BiC}$ and $\mathrm{CxiC}$ objects in the virtual environment. These values are compared with the values obtained from the physical environment. Further, formulas can be derived from the consistent values obtained from the virtual and physical environment.

\section{Conclusion}

This research work focuses on the behavior representation of a multidisciplinary product model by introducing Info-Chunk objects in the Information Content (IC). It started with the conversion of Info-Chunk entities into the Info-Chunk objects. Further, "InfoChunkLib" API is proposed based on the communication between the MAAD structure and RFLP structure in terms of $\mathrm{LiC}, \mathrm{BiC}$, and CxiC objects. Information Content (IC) is an application that imports InfoChunkLib API to handle and drive a multidisciplinary product model. The generated graph obtained from the IC evaluate the behavior of product components at various processes. The IC facilitate the HCI of multidisciplinary product model by initializing the parameters through the application. Info-Chunk objects provide necessary specification and knowledge representations to simulate the behavior of the complex multidisciplinary product model.

\section{Future Work}

A web server could be the next step for this research work, where a database is populated by the proposed API and a web application is used to access the IC. Dassault Systemes has implemented the RFLP structure in the CATIA V6 and 3DEXPERIENCE (3DXP) platforms for the multidisciplinary product model. Here, Dymola [16] is used to analyze the dynamic logical behavior and Modelica is used for logical and physical modeling of a product. The Java language and Modelica language are based on OOP concepts. Hence, API could be translated accordingly. The author could further update the API and database. Also, InfoChunkLib API can be extended and deployed in the IP. 


\section{Acknowledgment}

The author gratefully acknowledges his supervisor, Dr. Horváth László, for guidance while writing this article.

\section{References}

[1] L. Horváth and I. J. Rudas: Towards the Information Content-driven Product Model, Proceedings of the IEEE International Conference on System of Systems Engineering, Singapore, 2-4 June 2008, pp. 1-6

[2] L. Horváth and I. J. Rudas: Systems engineering in product definition, Proceedings of the IEEE $13^{\text {th }}$ International Symposium on Applied Machine Intelligence and Informatics (SAMI), Slovakia, 22-24 Jan. 2015, pp. $181-186$

[3] H. F. Krikorian: Introduction to object-oriented systems engineering.1, Journal of IT Professional, V(2), pp. 38-42, 2003

[4] L. Horváth and I. J. Rudas: Multilevel Abstraction Based Self Control Method for Industrial PLM Model, Proceedings of the IEEE International Conference on Industrial Technology, South Korea, 26 Feb.-1 March 2014, pp. $695-700$

[5] L. Horváth and I. J. Rudas: Active Driving Content in RFLP Structured Product Model, Recent Advances on Mechanics, Materials, Mechanical Engineering, and Chemical Engineering, MMMCE, Barcelona, 2015, pp. 123-131

[6] Peter Fritzson: Principles of Object-Oriented Modeling and Simulation with Modelica 3.3: A Cyber-Physical Approach, Wiley-IEEE Press, John Wiley \& Sons Inc, 2015, pp. 45-97

[7] Detterfelt, Jonas and Johansson, Gert: A UML Based Modeling Approach for Multi Domain System Products, Nordic Conference on Product Lifecycle Management - NordPLM, 2006, pp. 39-50

[8] John Stark: Product Lifecycle Management: $21^{\text {st }}$ Century Paradigm for Product Realisation, Springer-Verlag, London, 2011, pp. 10-25

[9] Yatish Bathla: Conceptual Models of Information Content for Product Modeling, Acta Polytechnica Hungarica, XV (2), 2018, pp. 169-188

[10] Ou Y.: On Mapping Between UML and Entity-Relationship Model, The Unified Modeling Language, Schader M., Korthaus A. (eds), Springer Nature, Switzerland, 1998, pp. 45-57

[11] Bernhard Thalheim: Entity-Relationship Modeling: Foundations of Database Technology, Springer-Verlag, New York, 2000, pp. 124-145

[12] L. Horváth, J. Fodor, I. J. Rudas: Manufacturing Aspect of the IBCA Structure for Active Knowledge Content Representation in Product Model, Journal of IFAC- PapersOnLine, 48(3), 2015, pp. 1616-1621 
[13] Yatish Bathla: Different types of process involved in the information content product model. In Proceedings of the IEEE $14^{\text {th }}$ International Symposium on Intelligent Systems and Informatics (SISY), 2016, pp. 99104

[14] L. Horváth and I. J. Rudas: Integrated Associative Modeling of Parts and their Machining Process by Features, Proceedings of the IEEE International Conference on Microelectronic Test Structures (ICMTS) conference, 2001, pp. 316-321

[15] Ian Sommerville: Software Engineering: $9^{\text {th }}$ edition, Addison-Wesley, Pearson Education \& Sons Inc, 2011, pp. 216-421

[16] Dassault Systemes AB: Dymola Dynamic Modeling Laboratory Getting started with Dymola. Dymola User Manual Volume 1, Dassault Syst emes, Lund, Sweden, 2013, pp. 23-48

[17] Yatish Bathla: Structured organization of Engineering Objects in the information content of the PLM system. In Proceedings of the IEEE $11^{\text {th }}$ International Symposium on Applied Computational Intelligence and Informatics (SACI), 2016, pp. 473-478

[18] L. Horváth and I. J. Rudas: Behavior and Design Intent Based Product Modeling, Acta Polytechnica Hungarica, 1(2), 2004, pp. 17-34

[19] Yatish Bathla: Info-Chunk driven RFLP Structure based Product Model for Multidisciplinary Cyber Physical Systems, Proceedings of the IEEE $16^{\text {th }}$ International Symposium on Intelligent Systems and Informatics (SISY), 2018, pp. 000327-000332

[20] L. Horváth and I. J. Rudas: Bringing up product model to thinking of engineer, Proceedings of the IEEE International Conference on Systems, Man and Cybernetics, 2008, pp. 1355-1360

[21] László Horváth: New methods on the way to intelligent modeling in computer integrated engineering. In Proceedings of the $36^{\text {th }}$ Annual Conference on IEEE Industrial Electronics Society (IECON), 2010, pp. 1359-1364 